Boise State University

ScholarWorks

$3-1-2015$

\title{
A Multiyear Dust Devil Vortex Survey Using an Automated Search of Pressure Time Series
}

Brian Jackson

Boise State University

Ralph Lorenz

Johns Hopkins University Applied Physics Laboratory 


\section{Journal of Geophysical Research: Planets}

\author{
RESEARCH ARTICLE \\ 10.1002/2014JE004712 \\ Key Points: \\ - A barometric sensor network \\ provides robust dust devil population \\ statistics \\ - Population statistics correlate with \\ season, time of day, and precipitation \\ - Gauging dust devil influence needs \\ population statistics, not just mean \\ values
}

Correspondence to:

B. Jackson,

bjackson@boisestate.edu

Citation:

Jackson, B., and R. Lorenz (2015) A multiyear dust devil vortex survey using an automated search of pressure time series, J. Geophys. Res. Planets, 120 doi:10.1002/2014JE004712.

Received 11 AUG 2014

Accepted 4 FEB 2015

Accepted article online 9 FEB 2015

\section{A multiyear dust devil vortex survey using an automated search of pressure time series}

\author{
Brian Jackson ${ }^{1,2}$ and Ralph Lorenz ${ }^{3}$ \\ ${ }^{1}$ Carnegie Institution for Science, Washington, District of Columbia, USA, ${ }^{2}$ Department of Physics, Boise State University, \\ Boise, Idaho, USA, ${ }^{3}$ Johns Hopkins University Applied Physics Laboratory, Laurel, Maryland, USA
}

\begin{abstract}
Dust devils occur in arid climates on the Earth and ubiquitously on Mars, where they likely dominate the supply of atmospheric dust and influence climate. Martian dust devils have been studied with a combination of orbiting and landed spacecraft, while most studies of terrestrial dust devils have involved manned monitoring of field sites, which can be costly both in time and personnel. As an alternative approach, we describe a multiyear in situ survey of terrestrial dust devils using pressure loggers deployed at El Dorado Playa in Nevada, USA, a site known for dust devil activity. Analogous to previous surveys for Martian dust devils, we conduct a posthoc analysis of the barometric data to search for putative dust devil pressure dips using a new automated detection algorithm. We investigate the completeness and false positive rates of our new algorithm and conduct several statistically robust analyses of the resulting population of dips. We also investigate possible seasonal, annual, and spatial variability of the putative dust devil dips, possible correlations with precipitation, and the influence of sample size on the derived population statistics. Our results suggest that large numbers of dips $(>1000)$ collected over multiple seasons are probably required for accurate assessment of the underlying dust devil population. Correlating long-term barometric time series with other data streams (e.g., solar flux measurements from photovoltaic cells) can uniquely elucidate the natures and origins of dust devils, and accurately assessing their influence requires consideration of the full distribution of dust devil properties, rather than average values.
\end{abstract}

\section{Introduction}

Dust devils are small-scale (few to many tens of meters) low-pressure vortices rendered visible by lofted dust. They have been observed to persist from minutes to hours and can travel kilometers, often carried by the ambient wind [Lorenz, 2013a]. On Earth, they are observed in arid locations primarily, where the ground is usually dry enough to provide a ready supply of dust [e.g., Balme and Greeley, 2006]. On Mars, they have been observed ubiquitously, both from the ground [Metzger et al., 1999] and from orbiting spacecraft [Cantor et al., 2006]. On both planets, dust devils contribute to the atmospheric aerosol content, sometimes increasing the dust content over the U.S. Southwest by more than an order of magnitude [Renno et al., 2004]. On Mars, dust devils may be the primary source for atmospheric dust, which plays a role in the radiative balance of the Martian atmosphere and, therefore, on the planet's meteorology [Basu et al., 2004]. Dust devils also seem to have lengthened the operating lifetime of Martian rovers by frequently cleaning their solar panels (http://mars.jpl.nasa.gov/mer/mission/status_opportunityAll.html\#sol3603). Since the dust supply from dust devils on both planets may be dominated by the seldom observed larger devils, it is particularly important to study the underlying distribution of dust devils, rather than focusing on the typical devil. Thus, elucidating the origin, evolution, and population statistics of dust devils is critical for understanding important terrestrial and Martian atmospheric properties and for in situ exploration of Mars.

While the pressure dips associated with dust devils have been recorded on Earth [e.g., Wyett, 1954; Lambeth, 1966; Sinclair, 1973], they are actually more systematically documented in studies of dust devils on Mars (e.g., by Mars Pathfinder: Murphy and Nelli, 2002; and by the Phoenix mission: Ellehoj et al., 2010), where landers have recorded meteorological parameters over long periods with a high enough cadence to detect small vortical structures. Most terrestrial meteorological records have cadence too low (canonically, $15 \mathrm{~min}$ ) to record dust devils, for which a sampling rate of $\sim 1 \mathrm{~Hz}$ or better is typically required.

Here we examine a continuous high-resolution pressure record to provide a census of vortices on Earth at El Dorado Playa near Boulder City, Nevada, a location known for dust devil activity [e.g., Pathare et al., 2010; 
Metzger et al., 2011]. Because the survey is made posthoc on the pressure time series, it is directly analogous to the Martian surveys. In addition to being a convenient measure of the overall intensity of a dust devil (as is also the case with other vortex motions such as tornadoes) [e.g., Karstens et al., 2010], the pressure dip may itself play a significant role in dust lifting [e.g., Balme and Hagermann, 2006]. As at Mars, such an approach detects pressure dips from all vortices without reference to whether they are dust laden or not. One feature initially noted in the data presented here is a diverse range of skewed or multipole signatures resulting from the cycloidal path of a devil nearby [Lorenz, 2013b], even for single-core vortices.

Lorenz and Lanagan [2014] performed a manual search of a subset of the data analyzed here, a month long measurement series (about 20 million data points) from three stations deployed during summer 2012, to identify about 650 discrete pressure dips that are interpreted as vortex signatures. For longer surveys, and for additional field sites, this manual approach will be somewhat laborious; and hence, in the present paper, we develop an automated search for these signatures.

\section{Instrumentation and Data Collection}

Previous terrestrial in situ dust devil studies involved field campaigns with personnel in attendance, which usually entails significant labor costs. Our approach has been to deploy small data acquisition systems which can operate for months without intervention. Recovery of a unit is required in order to retrieve its data, which are stored on board in flash memory, but the units are sufficiently inexpensive (compared with labor and transportation costs to deploy) that multiple units can be deployed, and, as long as a subset is recovered, the operation overall is cost effective.

The systems used [Lorenz, 2012a] are based around the Gulf Coast Data Concepts B1100 pressure logger, which combines a precision Bosch BMP085 pressure sensor (logged with a resolution of $1 \mathrm{~Pa}$ or $0.01 \mathrm{mb}$ ) with a microcontroller that logs the pressure data and housekeeping temperature as ASCII files on a $2 \mathrm{~GB}$ microSD flash memory card. The whole unit operates as, and its form factor resembles, a large USB memory stick, facilitating data transfer to a computer. As described in Lorenz [2012a], for this application the nominal single AA battery is replaced by a pair of alkaline $D$ cells, allowing unattended multimonth operation at sample rates of $2 \mathrm{~Hz}$ or more. The sensor and battery are installed in a plastic case, drilled to allow pressure equalization, and painted light brown to minimize visibility.

We deployed 15 loggers at six locales on El Dorado Playa over the course of about 2 years, and each logger is designated by a combination of letters and numbers in Table 1, such as S42, M01, etc. Table 1 also groups the loggers by location and shows the dates of operation and number of pressure dips detected. Figure 1 shows the approximate deployment locations (we have redacted the exact coordinates since observations are ongoing).

An initial deployment was made in April 2012. The strong temperature variations encountered by devices left on the playa surface in desert sunshine led to high-noise levels in the pressure history, due to improper temperature compensation of the pressure transducer readings, as discussed in Lorenz [2012a]. This issue was ameliorated by acquiring more frequent temperature readings, and data in June/July 2012 and thereafter have improved noise levels. In addition to the analysis here, June/July 2012 data from three stations were also analyzed by hand in Lorenz and Lanagan [2014]. One logger (designated P28) was deployed at the western margin of the playa, location A in Figure 1, while two others (P11 and P10) were deployed toward the eastern side near the station labeled D. Loggers at these locations were replaced during the course of observations reported here.

Continuous records at all locations by the same loggers over the entire observation period were not always possible since some loggers failed or were not retrieved. In other cases, the sequence of measurements at a particular station was maintained by different hardware loggers (e.g., logger P14 was deployed where P11 was previously deployed). Two stations (designated P14 and P23) were operated over the winter 2012-2013, although few events were expected. A wider suite of loggers was deployed in spring 2013 than in 2012. Logging at locations $D$ and $A$ was maintained to yield a comparison with the previous year. We deployed a cluster of stations in summer 2013 near the western side of the playa (A01, G01, M01, M02, and S42), all within about $50 \mathrm{~m}$ of each other near station $\mathrm{B}$.

Although the field site is one known to be hot and generally dry (hence the dust devil activity), the playa flooded in August 2012 and in July 2013. In the latter instance, water ingress into data logger boxes (which, 
Table 1. Sensor Record Location and Name, the Dates and Total Days Active, Latitude/Longitude, and Total Number of Pressure Dips Detected

\begin{tabular}{|c|c|c|}
\hline Location/Name & Dates (\# Days) & \# Dips \\
\hline 0. Location_A/P23 & $2012-11-27-2013-03-08(101)$ & 20 \\
\hline 1. " "/P28 & $2012-05-21-2012-06-11(21)$ & 139 \\
\hline 2. " "/" & 2012-06-11 - 2012-09-07 (88) & 88 \\
\hline \multirow[t]{2}{*}{ 3. "/S01 } & 2013-04-29 - 2013-07-17 (79) & $\underline{67}$ \\
\hline & Total Sensor Days: 289 & Total Dips: 314 \\
\hline 4. Location_B/Anemom1 & $2013-04-29-2013-07-19(81)$ & 86 \\
\hline 5. "/Geophone01 & $2013-04-29-2013-07-12(74)$ & 91 \\
\hline 6. " "/M01 & $2013-06-03-2013-07-14(41)$ & 22 \\
\hline 7. "/M02 & $2013-06-03-2013-07-20$ (47) & 26 \\
\hline \multirow[t]{2}{*}{ 8. " "/S42 } & 2013-06-03 - 2013-07-04 (31) & $\underline{28}$ \\
\hline & 274 & 253 \\
\hline 9. Location_C/SO2 & $2013-04-29-2013-07-14(76)$ & 84 \\
\hline 10. Location_D/P11 & $2012-05-21-2012-06-11(21)$ & 114 \\
\hline 11. " "/" & 2012-06-11 - 2012-07-12 (31) & 33 \\
\hline 12. $" / "$ & $2012-07-12-2012-08-22(41)$ & 25 \\
\hline 13. "/P14 & $2012-11-27-2013-04-24(148)$ & 48 \\
\hline 14. "/P16 & 2012-06-11 - 2012-09-04 (85) & 65 \\
\hline \multirow[t]{2}{*}{ 15. "/Radio } & 2013-06-03 - 2013-09-08 (97) & $\underline{66}$ \\
\hline & 423 & 351 \\
\hline 16. Location_E/S03 & $2013-04-29-2013-06-03(35)$ & 25 \\
\hline 17. Location_F/P10 & $2012-05-22-2012-06-11(20)$ & 32 \\
\hline 18. " "/" & 2012-06-11 - 2012-07-12 (31) & 21 \\
\hline \multirow[t]{2}{*}{ 19. " "/" } & 2012-07-12 - 2012-09-11 (61) & $\underline{17}$ \\
\hline & 102 & 70 \\
\hline
\end{tabular}

since they must monitor pressure, are not sealed) suspended data acquisition. Another hazard should be noted: in our April 2013 visit, a rattlesnake sought shade underneath our parked vehicle.

The total data set comprises 1200 station days, which exceeds by a factor of 13 the prior in situ terrestrial survey of 90 station days [Lorenz and Lanagan, 2014]. With a sampling of $2 \mathrm{~Hz}$, the data set amounts to $>252$ million measurements, motivating the application of automated methods.

\section{Data Conditioning and Pressure Dip Detection}

The data loggers record a pressure time series as a comma-delimited ASCII text file, with individual files typically spanning $12 \mathrm{~h}$. Over the course of a deployment, a logger might generate a few hundred such files. (By comparison, the available Martian pressure data sets have time extents ranging from several minutes to a day.) Our method for detecting dust devil pressure signals resembles that of

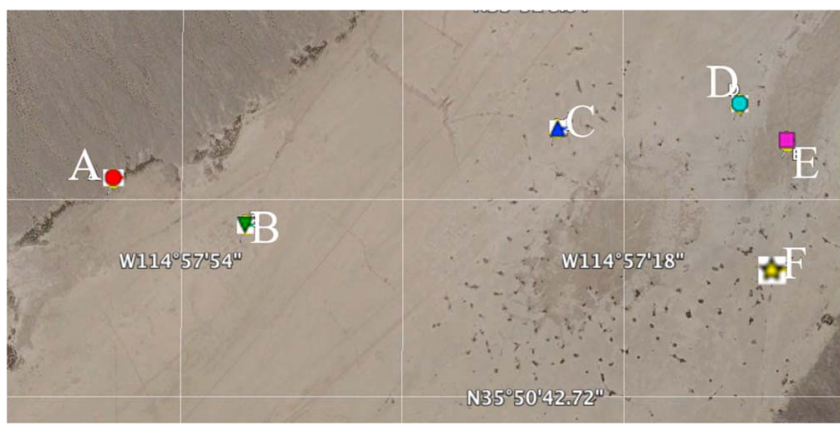

Figure 1. Google Earth map of the playa, with station locations marked. The symbols we use for each location throughout the paper are also shown, and the loggers deployed at each location are given in Table 1.
Wagstaff et al. [2006] and has some elements in common with other Martian dust devil searches [e.g., Ellehoj et al., 2010], namely using the difference between a short-term average and a long-term average [e.g., Ringrose et al., 2007]. This technique is referred to in the seismology literature as a "phase picker." However, we have much more data, and the data exhibit more variability, necessitating a more complex conditioning and detection process. Our initial detection algorithm proceeds as follows: 
1. We read in data text files and discard any corrupted ones. Corrupted files usually have only a few entries in them, spanning a time shorter than a few hundred seconds.

2. We discarded data points more than $10 \sigma$ away from the median value for a given data file. (Throughout the paper, $\sigma$ represents the standard deviation calculated as $1.4826 \times$ the median absolute deviation. This quantity is more robust against outliers than the usual standard deviation) [Bevington and Robinson, 2003]. This step typically removes only about $1 \%$ of the initial data.

3. We applied a mean boxcar filter to remove long-term trends possibly resulting both from meteorological and instrumental variability. We took a width $W=1000 \mathrm{~s}$ for the boxcar, and this choice has some modest influence on what signals we detect, as discussed in section 4.1. (Wagstaff et al. [2006] instead fit a polynomial as a smooth background against which to detect temporary excursions.) After the trends are removed, we add the data set mean back to the detrended data.

4. We calculated the median $\mu$ and $\sigma$ of the data within a window of width $W$ centered on time index $t_{\mathrm{i}}$. If the datum at this index, $d_{\mathrm{i}}$, lay below a chosen threshold number of $\sigma$ (i.e., negative but with a magnitude exceeding the threshold), we added it to the collection of points that may comprise dust devil dips. The data exhibit nonstationary noise, and so we needed to calculate the local $\sigma$ in a window $W$ around each point. Through inspection of the data and completeness tests discussed in section 4.1, we found that a $3 \sigma$ threshold provided a balance between recovering plausible dust devil signals and false positives.

5. We marched left and right from (before and after in time) an outlier point until we had a collection at least 100 points (or about $50 \mathrm{~s}$ ) that lay within $1 \sigma$ of $\mu$ to ensure that the entire feature had been captured. 6. Finally, we combined features that overlapped in time into a single feature.

With this collection of putative detections in hand, we applied a series of model analyses to further winnow the collection of dips:

1. We masked out the putative dips and reapplied the boxcar filter. We then linearly interpolated the filter signal across the dips to remove variability and to avoid distorting the pressure dips.

2. We fit a Lorentzian profile $L(t)$ to all the data points comprising a putative dip:

$$
L(t)=-\frac{\Delta P}{1+\left(\left(t-t_{0}\right) / \Gamma\right)^{2}}+B
$$

where $\Delta P$ is the magnitude of the pressure dip, $t_{0}$ the central time of the dip, $2 \Gamma$ the profile full width at half maximum (FWHM), and $B$ the baseline outside of the dip. (Physical arguments suggest that dust devils involve such a pressure structure [Rennó et al., 1998], and so such profiles have frequently been used for dust devil analyses) [Ellehoj et al., 2010]. We estimated the uncertainties on individual data points as the $\sigma$ value of points inside the window of width $W$ centered on a dip but not part of the dip itself. We used a Levenberg-Marquardt $\chi^{2}$-minimization scheme [Markwardt, 2009], and, for uncertainties on the fit parameters, we took the square root of the diagonal elements of the covariance matrix. Often, this fit produced $\chi_{v}^{2}>1\left(\chi_{v}^{2}\right.$ is the reduced $\left.\chi^{2}\right)$, suggesting that we may have underestimated the uncertainties, and so we rescaled the uncertainties (on all data points and on the model fit parameters) by $\sqrt{\chi_{v}^{2}}$ [Bevington and Robinson, 2003].

3. We redetermined the points constituting a dip by taking all points within 5 times the initial $\Gamma$ value of the initial $t_{0}$. (This step only shifted slightly the points constituting the putative dip, and we found that including more points often introduced background trends to the dip that confused the fitting algorithm.) We then applied the same model fit to these points. Fitting the model to these points produced the final best fit model parameters and uncertainties. We only included dips with $\Gamma$ values greater than 10 data points, typically $5 \mathrm{~s}$ but variable since the sampling frequency was not the same for all files.

4. We compared the final $\Delta P$ to the data point uncertainty to estimate the signal-to-noise ratio SNR for each dip and only retained those with SNR $>3$.

Figure 2 shows an example of the data conditioning and detection for one file and compares dips detected by the algorithm (blue curves) to those reported by Lorenz and Lanagan [2014] (red vertical lines).

\section{Results}

In this section, we first discuss estimates of the completeness rate and filtering out detections that may not be dust devil pressure signals. Then we discuss the results from our detection scheme and the distribution of model fit parameters for our detected dips. 


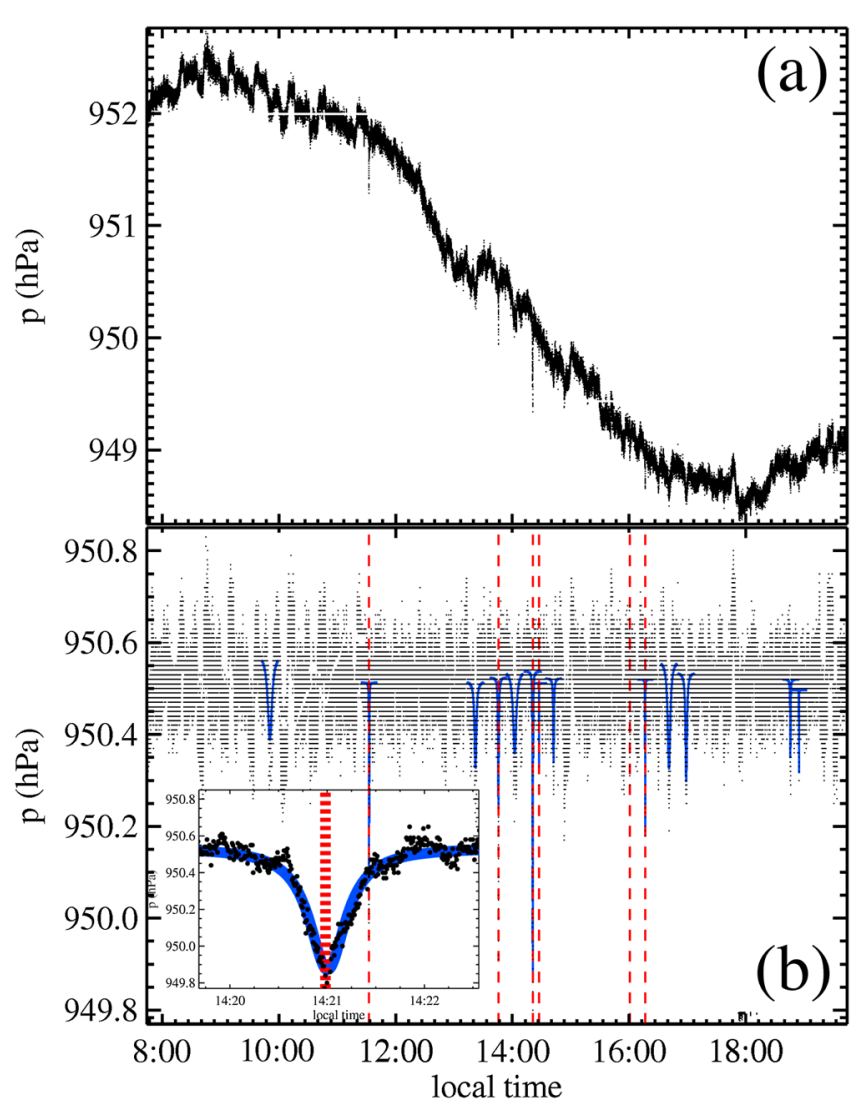

Figure 2. Pressure variations (in hectoPascal, $\mathrm{hPa}$ ) versus local time for one data file. (a) The raw data and (b) the detrended data. Blue curves show model fits for those dips that pass our detection thresholds, while red vertical lines show the dips reported for this file in Lorenz and Lanagan [2014]. The inset zooms in on the dip just after 14:00. The striping in the data results from discretization of the measurements to the nearest $\mathrm{Pa}$.

\subsection{Completeness and Filtering} Spurious Detections

To determine the algorithm's ability to recover known dips (completeness) and how often it may be fooled by spurious signals (false positives), we compared our detections to those generated by hand from Lorenz and Lanagan [2014]. Figure 2 compares a few detections from our automated algorithm to those reported by Lorenz and Lanagan [2014]. For this data file, the algorithm detected five of six dips reported previously and generated an additional eight possibles not reported.

To test our choices of $W$ and SNR threshold on the completeness rate, we considered values of $W$ ranging from 10 up to $1000 \mathrm{~s}$ and SNR thresholds between 3 and 10. For each W/SNR combination, we calculated the completeness by determining how many "Class 1" (certain dust devil) dips we recovered and divided by the total number reported in Lorenz and Lanagan [2014]. If the midtime $t_{0}$ for a detected dip lay within 3 FWHMs of the time reported for a detection in Lorenz and Lanagan [2014], we considered that dip recovered. Based on these calculations, we took $W=1000 \mathrm{~s}$ and $\mathrm{SNR}>3$, giving a

completeness greater than 75\%. We note that the Class 1 detections in Lorenz and Lanagan [2014] all had SNR $\geq 4$, while their "possible" detections had SNR between 3 and 8 . All the Class 1 detections also had pressure dips of $0.2 \mathrm{hPa}$ or more.

This method of data conditioning means we likely distort or do not detect longer duration pressure dips representing large and/or slow-moving dust devils. For example, a dip lasting nearly $1000 \mathrm{~s}$ would simply be treated as a background fluctuation and either be filtered out or contribute to the estimate of $\sigma$. However, any detection scheme will overlook signals that differ significantly from the expected signal-that is the purpose of a detection scheme.

Unfortunately, determining a false positive rate is more difficult since dips found by the algorithm but not by Lorenz and Lanagan [2014] may or may not be real dust devils. (Lorenz and Lanagan [2014] do not report a completeness rate.) In fact, using the $W$ value and SNR threshold given above, the algorithm reported nearly 70\% more dips than Lorenz and Lanagan [2014] using the same data set, but visual inspection suggests many were not true dust devil dips_-most are unexpectedly shallow. Figure 3a compares the average dip reported by Lorenz and Lanagan [2014] (black) to the average one found here but not previously reported (red). Note that each average profile shown has been normalized to its own best fit pressure depth so they can all appear on the same scale. Figure $3 \mathrm{~b}$ shows the ratio of $\Delta P$ to FWHM for the same two classes of dip-those not previously reported tended to cluster at small values of this ratio. Assuming that many of these previously unreported dips are spurious detections, we calculated $\sigma$ for the $\Delta P$ to FWHM ratio for this collection of dips (about $0.034 \mathrm{hPa} / \mathrm{s}$ ) and dropped dips with values of this ratio $<\sigma$. The blue curve in Figure 3a shows the average for the remaining previously unreported dips, which closely resembles the 

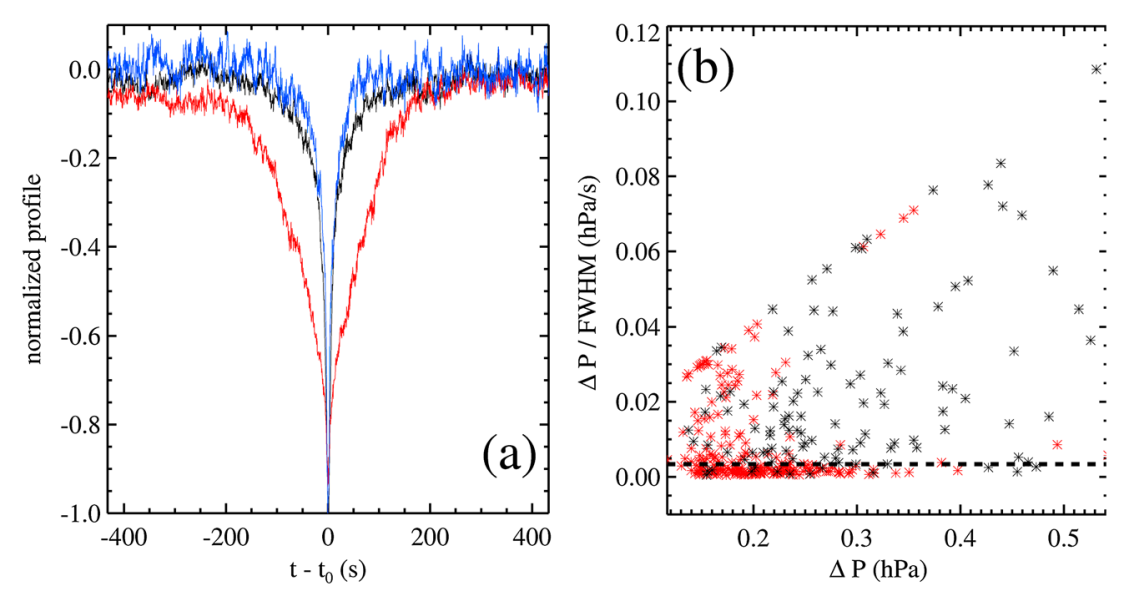

Figure 3. Normalizing each pressure dip individually by its best fit $\Delta P$ value, (a) the resulting average profile for dips reported in Lorenz and Lanagan [2014] (black) and dips found here but not previously reported (red). (b) The distribution of $\Delta P$ versus $\Delta P / F W H M$ for dips previously reported (black stars) and those not (red stars). Removing dips below the dashed black line in Figure 3b produces the average, normalized profile in blue in Figure 3a.

black curve, suggesting we successfully removed most spurious dips. We applied this same filtering to all the following dip analyses reported here.

\subsection{Dip Statistics}

\subsubsection{Overall Statistics}

Figure 4 shows the resulting distribution of fit parameters for the combined data set. Error bars are suppressed for clarity but are typically $0.01 \mathrm{hPa}$ for $\Delta P, 3 \mathrm{~s}$ for FWHM, and $0.5 \mathrm{~s}$ for $t_{0}$. Our analysis produced a large collection of putative dips with FWHM $\lesssim 5 \mathrm{~s}$, not shown in Figure 4. These FWHM values represent the smallest values allowed for $2 \Gamma$ by our modeling fitting, $5 \times$ the sampling interval for a data file. Either they are spurious detections (inspection of some suggests not), or there really are such short duration dips, representing small/fast-moving dust devils. However, additional work is required to adequately assess this population, so we conservatively include only dips with FWHM $>10$ s-reflected in Figure 4. In total, we ended up with 1667 dips. To facilitate comparison with other data sets, Figure 5 shows a scatterplot of FWHM versus $\Delta P$ with linear axes - we have zoomed in on the highest density region. The shaded, red region shows where we have excluded dips for being too shallow. It is not obvious that dips in that region are anomalous, but additional analysis and field work are probably required for a complete understanding.

It is worth noting that, since pressure loggers were often deployed within close proximity of one another, passage of a single dust devil may have registered a dip on each of several loggers at nearly the same time. However, additional data are required to distinguish these cases (e.g., the prevailing wind direction), and so we make no effort to for this study.

As discussed by Lorenz [2011], the choices of bin size and number in generating histograms of dust devil populations can significantly influence the results since these choices can affect a histogram's overall shape. We used Knuth's algorithm (http://www.astroml.org/user_guide/density_estimation.html) to generate the histograms in Figure 4, which employs a Bayesian framework, maximizing the posterior probability that a histogram with a given uniform bin size represents the data set under consideration [Knuth, 2006]. This approach to binning has the advantage that the bin sizes are objectively determined and tailored to the data. For Figure 4, the algorithm gives bin sizes of $0.09,0.07$, and $55 \mathrm{~min}$ for $\log (\mathrm{FWHM}), \log (\Delta P)$, and $t_{0}$, respectively. Alternative Bayesian binning methods have been proposed [e.g., Scargle et al., 2013], and expanding on Lorenz [2011] to investigate optimal binning choices will be the subject of future work.

Referring back to the results, Figure 4 indicates an abrupt increase in activity about 6 A.M., with a less dramatic drop-off after 6 P.M. Dust devils appear to be most common in the early afternoon, between 1 and 2 P.M., and the early afternoon sees some of the deepest pressure dips, completely consistent with previous studies [Balme and Greeley, 2006]. There also appear to be detectable pressure dips before sunrise and after sunset. These detections represent a small fraction of the total, and it is not clear whether they are spurious (again, inspection suggests not) or are associated with meteorological phenomena other than dust devils. Figure 5 shows that the distribution of $\Delta P$ values has a clear peak, with a median of about $0.24 \mathrm{hPa}$ and 16 th 

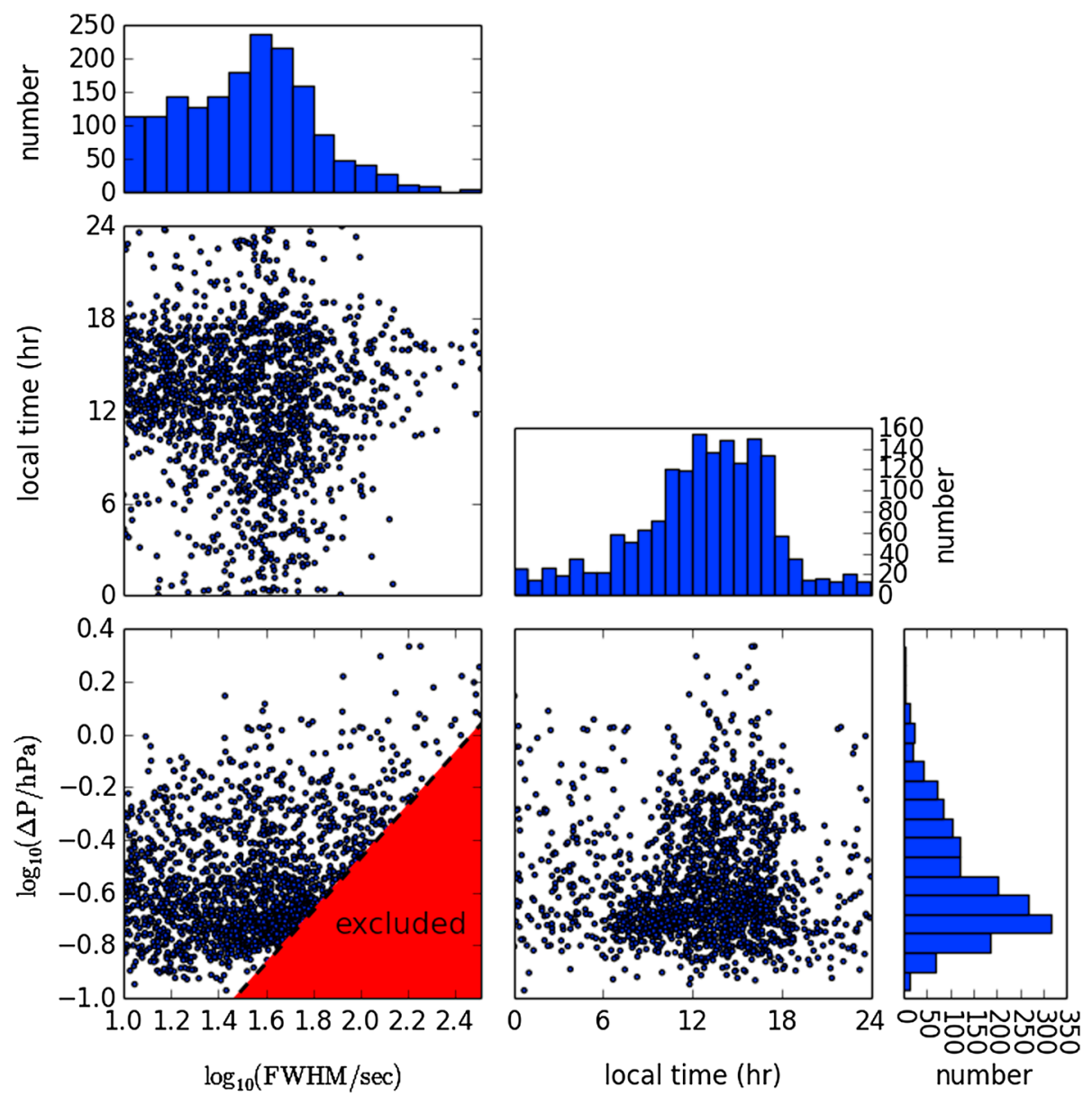

Figure 4. Distribution and histograms for $\Delta P$, full width at half maximum ( $F W H M=2 \Gamma)$, and local time $t_{0}$ of dips. The shallow dips excluded are indicated by the red region in the bottom right-hand panel.

and 84th percentiles (analogous to $\pm 1 \sigma$ for nonnormal distributions) of $0.18 \mathrm{hPa}$ and $0.48 \mathrm{hPa}$, respectively. FWHM values are broadly distributed, with a median of $34.5 \mathrm{~s}$ and 16th and 84th percentiles of $15.8 \mathrm{~s}$ and 59.4 s, respectively.

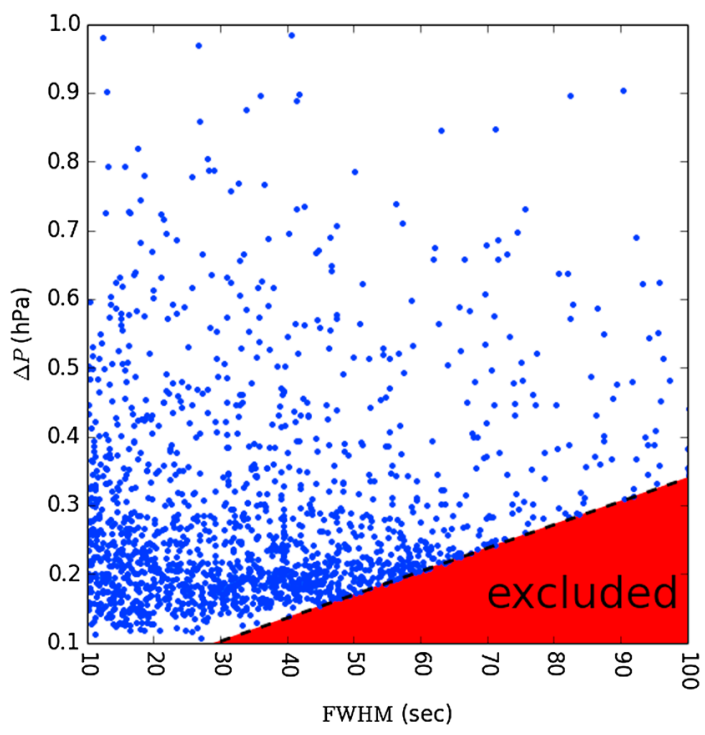

Figure 5. Similar to 4 except with linear axes.
The total number of dips recovered by our algorithm significantly exceeds the number of dust devils considered by Lorenz [2012b] and therefore allows a more robust determination of pressure dip statistics, in particular which distribution function provides the best fit. Lorenz [2011] and others have pointed out that different dust devil formation processes can give rise to different populations, and so, in principle, the population statistics can elucidate the origins of dust devils.

Figure 6a illustrates the $\Delta P$ histogram with bin sizes for $\Delta P$ of $0.03 \mathrm{hPa}$ determined by Knuth's algorithm. $Y$ error bars show Poisson uncertainties, and $X$ error bars show bin width. We compare a power law $\left(N=b(\Delta P / h P a)^{p}\right.$, solid red line) to an exponential fit ( $N=N_{0} e^{\Delta P / \lambda}$, dashed green line). The number of dips with $\Delta P$ below the histogram peak (at $0.24 \mathrm{hPa}$ ) drops off, possibly due to poor 

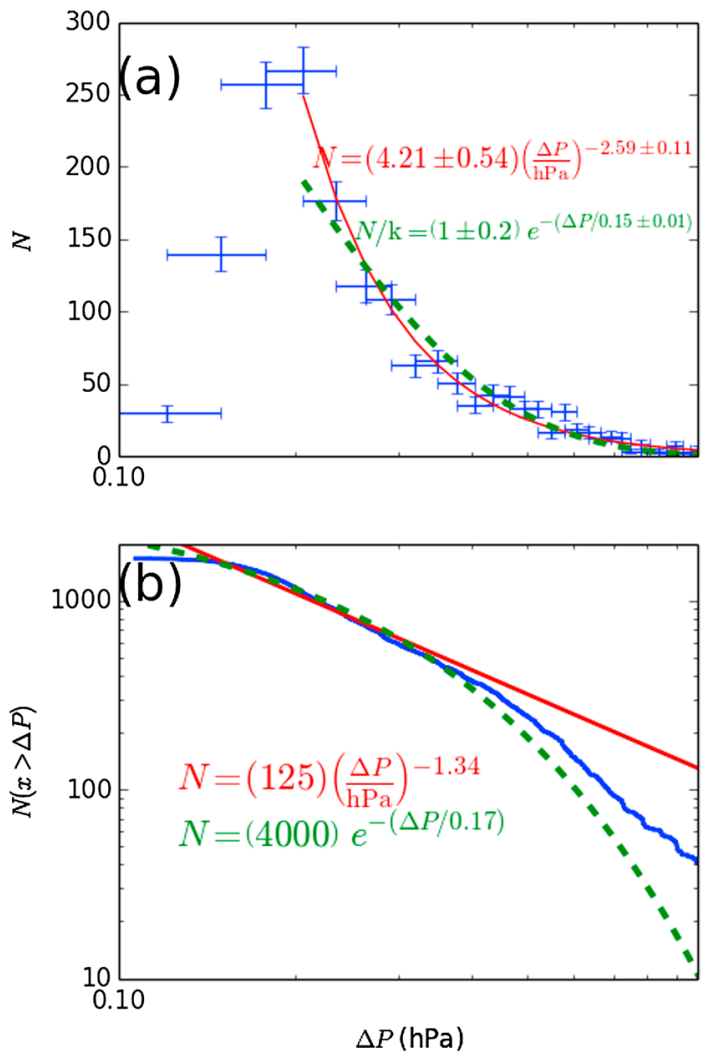

Figure 6. $\Delta P$ histogram, along with power law (solid, red line) and exponential (dashed, green line) fits and corresponding fit parameters. Bin size is $0.03 \mathrm{hPa}$. The standard deviations of the posterior distributions returned by the Markov chain Monte Carlo (MCMC) analysis are used for uncertainties on the model parameters.
SNR, so we elected only to fit the power law for $\Delta P>0.24 \mathrm{hPa}$, leaving $1097 \mathrm{dips}$. The best fit parameters for both models are shown in the figure (power law on top, exponential on bottom).

To generate the fits, we dropped bins with fewer than two members and then applied a standard Markov chain Monte Carlo (MCMC) algorithm [Foreman-Mackey et al., 2013]. We checked for convergence by requiring the correlation length of each chain to be at least 10 times shorter than the chains themselves (https://github.com/dfm/ acor). We took a burn-in length equal to a tenth of the total chain length. Finally, we took the best fit values for both models as the 50th percentiles of the samples in the marginalized distributions and approximated the uncertainties as the distribution standard deviation. The $\chi_{v}^{2}$ value for the power law fit is 1.76 and that for the exponential is 3.54, indicating the power law is clearly preferred. $\chi_{v}^{2}>1$ may mean we underestimated bin uncertainties somewhat but does not affect intercomparison of the models since they both involve the same data.

Figure $6 \mathrm{~b}$ illustrates a cumulative histogram, with the same functions fit using the same algorithm. However, we do not report uncertainties on the fit parameters since we do not have error bars for the data points in the cumulative histogram. In this case, a $\chi^{2}$ comparison favors the exponential function because all data points are weighted equally without uncertainties, and the exponential

does a better job of fitting the points with larger $\Delta P$. Note also that the fit parameters for the cumulative histogram do not agree with what we would expect if we integrated the functional fits for the differential histograms. This exercise shows the influence of different binning and weighting on the resulting fits-slightly different binning produces completely different results.

Note that one could fit a log normal or Weibull or other distribution to the recovered dips. However, it is not clear how much the fall off below $0.24 \mathrm{hPa}$ is due to poor detection efficiency, and how much to an intrinsically small number of events. Lorenz and Lanagan [2014] noted that the Deardorff convection speed associated with strong desert heating corresponds to a pressure fluctuation of about $0.04 \mathrm{hPa}$.

These results generally agree with previous studies. Lorenz and Lanagan [2014] found a cumulative slope for the manually detected P28 June/July 2012 data of -1.5 , corresponding to a differential exponent of -2.5 and in agreement with our results for a differential histogram. Fitting data from the Mars Phoenix lander with a power law, Lorenz [2012b] derived an index of $-2.20 \pm 0.47$, again in agreement with our results. However, in the same study, a power law fit to Pathfinder data produced an index $-1.56 \pm 0.49$, about $2 \sigma$ discrepant with our results. Their fit to the 19 dips reported by Lambeth [1966] gave $-0.76 \pm 0.74$.

Two important differences between ours and other analyses may contribute to disagreement: different histogram binning and a different population size. Lorenz [2011] considered the influence of the former, so here we investigate the latter. For this purpose, we randomly selected some number of pressure dips from the full population until we reached a desired number of sample points and then calculated a differential histogram using the same binning as before. Employing a Levenberg-Marquardt algorithm to facilitate the calculation, we then fit that histogram with a power law to determine the dependence of the index on sample size and repeated this process 1000 times for each desired number of dips from 20 up to 1667 dips. For this calculation, we initially retained all dips, regardless of their $\Delta P$ value and dropped dips 


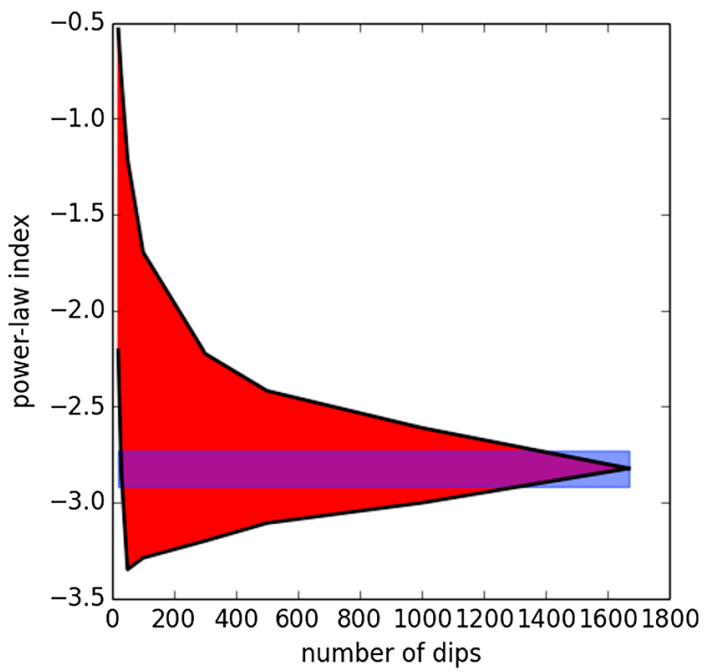

Figure 7. Dependence of the best fit power law index on number of dips in a population. The red, shaded region shows the $1 \sigma$ uncertainties for the index using a subset of the full population, while the blue, shaded region shows the best fit index and uncertainties produced by analyzing the full population.

with $\Delta P$ below the maximum in the histogram, exactly as we did for the full population of dips. Figure 7 shows the results - the red, shaded region shows the $1 \sigma$ range of indices, while the blue, shaded region shows the range of uncertainties for the index when we analyze all dips. The figure shows we would have to find between 1200 and 1400 dips before the best fit index approximates the correct, underlying value. For example, the small sample size from Lambeth [1966] (with 19 dips) as compared to ours can easily explain the discrepancy between power law indices. This result suggests that large numbers of pressure dips are probably required for an accurate assessment of the population statistics.

\subsubsection{Seasonality and Influence} of Precipitation

Figure 8 shows the occurrence of dust devils through the observational seasons, from late May to September 2012 and then again from December 2012 to early June 2013. For this plot, we have only included dips with $\Delta P>0.24 \mathrm{hPa}$ to mitigate the possibly poor detection statistics for the smaller peaks. Figure 8 also shows precipitation data from a weather station deployed by the Community Environmental Monitoring Program (http://cemp.dri. edu/) near Boulder City, Nevada, and about 16 km from our survey location. We applied the Knuth binning algorithm separately to the dip counts and precipitation data and took the smallest bin size, giving bins about 14 days wide.

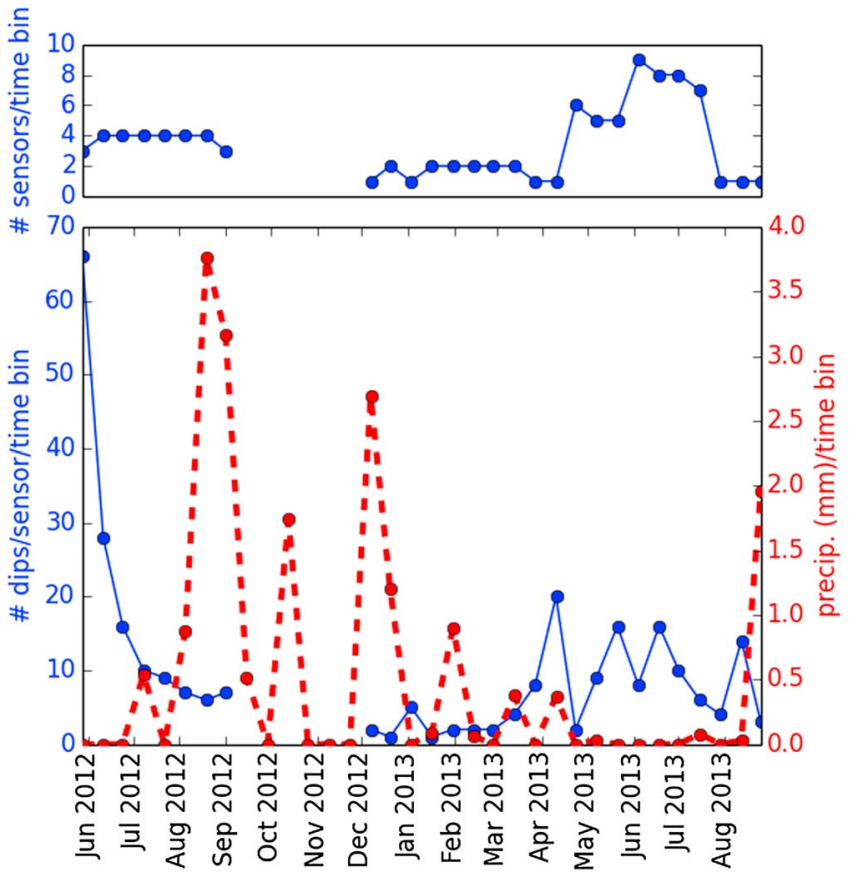

Figure 8. (top) The total number of sensors deployed during a time bin. As indicated in the text, we deployed more sensors in 2013 than in 2012. (bottom) The blue, solid line shows the number of pressure dips per sensor (there are no observations between October 2012 and late November 2012-see Table 1). The red dashed line shows the precipitation at a weather station about $16 \mathrm{~km}$ from our survey location. Bins are 14 days wide.
The seasonality of dust devils is obvious in the figure, with the summer occurrence rate significantly greater than those of other seasons. The average number of dips per sensor seems to have been much larger in summer 2012 than in summer 2013, the cause of which is not clear. Interestingly, though, observations record no precipitation between mid-March and mid-July in 2012, whereas 2013 saw precipitation events as late as May, possibly suppressing dust devil numbers that summer. Dust devil formation is expected to be suppressed for an extended period after a precipitation event, as the playa may be damp or even flooded for weeks, depending on the evaporation rate. In addition to the suppression of dust lifting (note that airborne dust may amplify dust devil intensity via injection of heat into the vortex) [e.g., Lorenz and Myers, 2005; Fuerstenau, 2006], the increased thermal inertia of the hard and/or damp playa will reduce the intensity of surface heating 

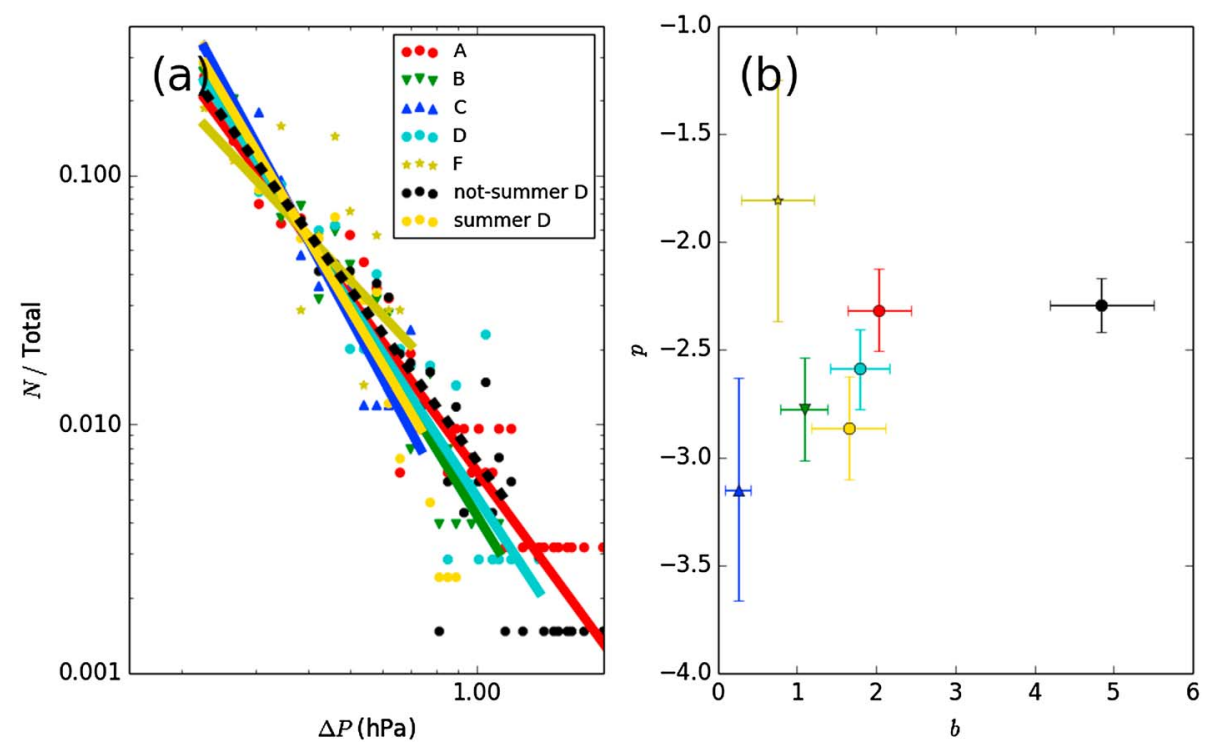

Figure 9. (a) $\Delta P$ histogram and best fit power law scale $b$ and index $p$ for each logger location. Note that each histogram is normalized to the total number of dips to allow us to show all data sets on the same plot. (b) Distribution of best fit power law parameters for each logger location, same symbols as Figure 9a. As before, model parameter uncertainties come from the posterior distributions.

that drives all convective vortices, whether dust laden or not. Figure 8 suggests an anticorrelation between precipitation and dip occurrence, but a causal link is not clear.

An important caveat here is that the precipitation record shown may or may not reflect rainfall at El Dorado Playa since rainfall in summer storms can be highly localized, even on the scale of the $16 \mathrm{~km}$ distance from the playa to the Boulder City weather station.

The observed variability in dip frequency is also influenced by the number of sensors deployed during an observational span, reflected in Figure 8 (top). For example, in some cases additional sensors were deployed at locations that saw few dips, so the average number of dips per sensor would drop, even if the underlying dip frequency had not changed. This effect may have influenced the drop in dip number in June 2013, when nearly twice as many sensors were deployed as during the previous observation span.

4.2.3. Comparison Between Logger Locations

Since the loggers span a range of locations and monitoring times, they may have recorded different populations of dust devils. Table 1 clearly shows that some loggers recorded more dips than others, so we also analyzed the data set for each logger location individually. We first created a histogram of each location's $\Delta P$ values, exactly as we did for the full combined data set (using exactly the same bins), and fit each histogram with a power law, employing the same MCMC analysis as above. Figure 9 shows the resulting histograms and model fit parameters. With only 25 dips detected in the final reckoning, location E provided poor statistics for this analysis, and so we excluded it here.

Inspection of Figure 9a shows reasonable agreement between histograms for most locations, and Figure $9 \mathrm{~b}$ shows that the corresponding power law indices all nearly agree to within uncertainties (calculated from standard deviations for the posterior distributions returned by the MCMC model fit). The indices for locations $\mathrm{C}$ and $\mathrm{F}$ differ by about $1.7 \sigma$, indicating marginal disagreement. However, these locations also have the fewest recorded dips ( 84 and 70 , respectively), which might explain why their power law fits stand out.

Seasonal variability seems to contribute to differences in dip populations since not all loggers recorded during the same time spans. To investigate this effect, we split the dips recorded at location $D$ (with the most dips) into those recorded during the summers 2012 and 2013 and those recorded during other seasons. We applied the same binning and power law fitting, and the results are also shown in Figure 9 , as black and gold symbols and lines. Figure 9 shows clear disagreement between the fit parameters for summer and not summer dips recorded at $D$, suggesting that we can, indeed, see seasonal influences. There are many more large $\Delta P$ dips at location $D$ outside of the summer than during the summer, as reflected in the larger $b$ and 


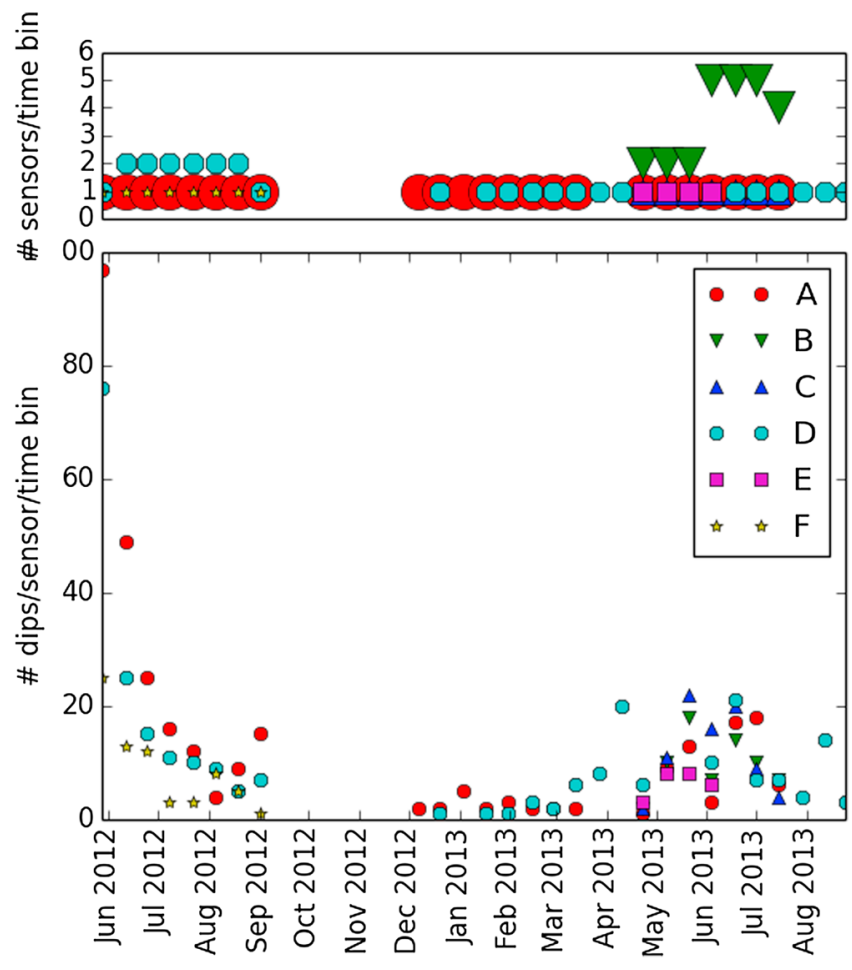

Figure 10. Number of pressure dips for each logger location. Bins are 14 days wide. $p$ values for "not summer D." It may be that stronger heating in summer yields many more small vortices since the near-surface air destabilizes more quickly, or it may be that ambient winds are stronger in winter, preferentially allowing only the largest vortices to form and persist.

We also plot the occurrence rate by location in Figure 10, analogous to Figure 8 (but without precipitation). The contrast between summers 2012 and 2013 is particularly pronounced in this figure-all three active locations saw a larger dip frequency in summer 2012 than in summer 2013. Several previous studies have considered the connections between dust devil occurrence and spatial/ temporal conditions [e.g., Sinclair, 1969; Snow and McClelland, 1990], and more thoroughly probing those connections in our data sets will be the subject of future work.

\section{Discussion and Conclusions}

We have reported the largest barometric dust devil vortex survey data set to date. This is not only the largest dust devil data set for Earth but also for Mars which until recently had better fixed-station surveys than for Earth. This large data set results from new field data acquisition techniques and the application of data analysis methods.

Among other key results, our study highlights the importance of studying dust devil population statistics and cautions against characterizing dust devils by average values. For example, based on analog experiments designed to mimic terrestrial conditions, Neakrase et al. [2006] suggested that the dust flux generated by a dust devil $F$ goes exponentially with the depth of the pressure well $\Delta P$. Using Figure 4 from that study and the average $\Delta P$ from our survey, $0.4 \mathrm{hPa}$, to calculate an average flux gives an estimate $(\langle F\rangle=F(\langle\Delta P\rangle))$ more than 1000 times smaller than integrating the flux against the dust devil population we retrieve here $\left(\langle F\rangle=\int F n(\Delta P) \mathrm{d}(\Delta P) / \int n(\Delta P) \mathrm{d}(\Delta P)\right)$. Not surprising, since an exponential dependence on $\Delta P$ means the average flux is dominated by the rare vortices with the lowest pressure cores.

We find that the population function of the pressure dips we detect peaks at around $0.2 \mathrm{hPa}$, perhaps suggesting this is the limit of efficient detection with the noise performance of the pressure sensors used, or it may represent the minimum amplitude of pressure dip associated with vortices under field conditions. The distribution function above this value appears to be well described by a power law with an exponent between -2 and -3 , rather similar to previous analyses. We note a fall off at the larger vortex end of the distribution that may be real or may be a result of finite sample size. The exponent appears to change with season, with summer having proportionately more abundant small vortices. Future work will examine the relationship of ambient parameters (heating and wind) to the population function.

While the exponents are broadly the same across the playa, the overall abundance of vortices is not. Notably, vortices appear more frequently throughout most of our study at station A, at the western margin of the playa. This may be a result of southeasterly winds giving this station a longer fetch, allowing vortices to develop across the playa. This may also explain the more abundant detections at stations $C$ and $D$ relative to E and $F$. 
We observe a large difference between June 2012 and June 2013: the latter saw about a factor of 4 fewer vortices at all stations. Thus, there is substantial interannual variability-a single observing campaign cannot provide a definitive estimate of the long-term average population (as might be desired to compute dust fluxes, for example). We also see a decline in vortex encounter rates after rainfall events. We note that the vortex detection rate does not fall to zero outside of the summer, although the dip population is statistically distinct from the summer population. Similar to Ellehoj et al. [2010], we also observed dips during the night, which may be associated with meteorological phenomena other than dust devils.

As part of future work, we plan to correlate several kinds of meteorological data, which will improve our understanding of the variety of pressure signatures for dust devils. For example, if dust devils are robustly detected in time-lapse imaging of a study region as barometric data are collected, we could build a library of dust devil pressure signals to improve detections, similar to waveform correlation techniques used in seismology [Gibbons and Ringdal, 2006].

\section{Acknowledgments}

B.J. acknowledges travel support from the Carnegie Department of Terrestrial Magnetism. The work of R.L. was funded by NASA through the Mars Fundamental Research Program grant NNX12AI04G. Thoughtful reviews from Asmin Pathare, Jim Murphy, and another referee significantly improved this article. Our barometric data are available at http://www.astrojack.com/ research/.

\section{References}

Balme, M., and R. Greeley (2006), Dust devils on Earth and Mars, Rev. Geophys., 44, RG3003, doi:10.1029/2005RG000188.

Balme, M., and A. Hagermann (2006), Particle lifting at the soil-air interface by atmospheric pressure excursions in dust devils, Geophys. Res. Lett., 33, L19S01, doi:10.1029/2006GL026819.

Basu, S., M. I. Richardson, and R. J. Wilson (2004), Simulation of the Martian dust cycle with the GFDL Mars GCM, J. Geophys. Res., 109, E11006, doi:10.1029/2004JE002243.

Bevington, P. R., and D. K. Robinson (2003), Data Reduction and Error Analysis for the Physical Sciences, McGraw-Hill, Boston.

Cantor, B. A., K. M. Kanak, and K. S. Edgett (2006), Mars orbiter camera observations of Martian dust devils and their tracks (September 1997 to January 2006) and evaluation of theoretical vortex models, J. Geophys. Res., 111, E12002, doi:10.1029/2006JE002700.

Ellehoj, M. D., et al. (2010), Convective vortices and dust devils at the Phoenix Mars mission landing site, J. Geophys. Res., 115, E00E16, doi:10.1029/2009JE003413

Foreman-Mackey, D., D. W. Hogg, D. Lang, and J. Goodman (2013), EMCEE: The MCMC hammer, Publ. A. S. P, 125, 306-312.

Fuerstenau, S. D. (2006), Solar heating of suspended particles and the dynamics of Martian dust devils, Geophys. Res. Lett., 33, L19S03, doi:10.1029/2006GL026798.

Gibbons, S. J., and F. Ringdal (2006), The detection of low magnitude seismic events using array-based waveform correlation, Geophys. J. Int., 165, 149-166.

Karstens, C. D., T. M. Samaras, B. D. Lee, W. A. Gallus, and C. A. Finley (2010), Near-ground pressure and wind measurements in tornadoes* Mon. Weather Rev., 138, 2570-2588.

Knuth, K. H. (2006), Optimal data-based binning for histograms. ArXiv Physics e-prints.

Lambeth, R. L. (1966), On the measurement of dust devil parameters, Bull. Am. Meteorol. Soc., 47, 522-526.

Lorenz, R. (2011), On the statistical distribution of dust devil diameters, Icarus, 215, 381-390.

Lorenz, R. D. (2012a), Observing desert dust devils with a pressure logger, Geosci. Instrum. Method Data Syst. Discuss., 2, $477-505$.

Lorenz, R. D. (2012b), Power law distribution of pressure drops in dust devils: Observation techniques and Earth-Mars comparison, Planet. Space Sci., 60, 370-375.

Lorenz, R. (2013a), The longevity and aspect ratio of dust devils: Effects on detection efficiencies and comparison of landed and orbital imaging at Mars, Icarus, 226, 964-970.

Lorenz, R. D. (2013b), Irregular dust devil pressure drops on Earth and Mars: Effect of cycloidal tracks, Planet. Space Sci., 76, 96-103.

Lorenz, R. D., and P. D. Lanagan (2014), A barometric survey of dust-devil vortices on a desert playa, Boundary Layer Meteorol., 153, $555-568$.

Lorenz, R. D., and M. J. Myers (2005), Dust devil hazard to aviation: A review of US air accident reports, J. Meteorol., 30, 178-184.

Markwardt, C. B. (2009), Non-linear least-squares fitting in IDL with MPFIT, in Astronomical Data Analysis Software and Systems XVIII. Vol. 411 of Astronomical Society of the Pacific Conference Series, edited by D. A. Bohlender, D. Durand, and P. Dowler, pp. 251, Astronomical Society of the Pacific, San Francisco, Calif.

Metzger, S. M., J. R. Carr, J. R. Johnson, T. J. Parker, and M. T. Lemmon (1999), Dust devil vortices seen by the Mars Pathfinder Camera, Geophys. Res. Lett, 26, 2781-2784

Metzger, S. M., M. R. Balme, M. C. Towner, B. J. Bos, T. J. Ringrose, and M. R. Patel (2011), In situ measurements of particle load and transport in dust devils, Icarus, 214, 766-772.

Murphy, J. R., and S. Nelli (2002), Mars Pathfinder convective vortices: Frequency of occurrence, Geophys. Res. Lett., 29(23), 2103, doi:10.1029/2002GL015214.

Neakrase, L. D. V., R. Greeley, J. D. Iversen, M. R. Balme, and E. E. Eddlemon (2006), Dust flux within dust devils: Preliminary laboratory simulations, Geophys. Res. Lett., 33, L19S09, doi:10.1029/2006GL026810.

Pathare, A. V., M. R. Balme, S. M. Metzger, A. Spiga, M. C. Towner, N. O. Renno, and F. Saca (2010), Assessing the power law hypothesis for the size-frequency distribution of terrestrial and Martian dust devils, Icarus, 209, 851-853.

Renno, N. O., et al. (2004), MATADOR 2002: A pilot field experiment on convective plumes and dust devils, J. Geophys. Res., 109, E07001, doi:10.1029/2003JE002219.

Rennó, N. O., M. L. Burkett, and M. P. Larkin (1998), A simple thermodynamical theory for dust devils, J. Atmos. Sci., 55, $3244-3252$.

Ringrose, T. J., M. R. Patel, M. C. Towner, M. Balme, S. M. Metzger, and J. C. Zarnecki (2007), The meteorological signatures of dust devils on Mars, Planet. Space Sci., 55, 2151-2163.

Scargle, J. D., J. P. Norris, B. Jackson, and J. Chiang (2013), Studies in astronomical time series analysis. Vl. Bayesian block representations, Astrophys. J., 764, 167.

Sinclair, P. C. (1969), General characteristics of dust devils, J. Appl. Meteorol., 8, 32-45.

Sinclair, P. C. (1973), The lower structure of dust devils, J. Atmos. Sci., 30, 1599-1619.

Snow, J. T., and T. M. McClelland (1990), Dust devils at White Sands Missile Range, New Mexico: 1. Temporal and spatial distributions, J. Geophys. Res., 95, 13,707-13,721.

Wagstaff, K. L., R. Castano, and S. Chien (2006), Real-time, in-situ detection of dust devils using pressure sensors, AGU Fall Meeting Abstracts, A3.

Wyett, R. E. (1954), Pressure drop in a dust devil, Mon. Weather Rev., 82, 7-8. 